\title{
GENERIC PROPERTIES OF CONTRACTION SEMIGROUPS AND FIXED POINTS OF NONEXPANSIVE OPERATORS
}

\author{
F. S. DE BLASI AND J. MYJAK
}

\begin{abstract}
Let $\Omega$ be a nonempty, closed, bounded and starshaped subset of a Banach space $X$. It is shown that most (in the Baire category sense) differential equations $\boldsymbol{u}^{\prime}+A \boldsymbol{u}=0$ do have a unique asymptotic equilibrium point. Here $A: \Omega \rightarrow X$ is supposed to be a nonlinear, continuous, bounded and accretive operator satisfying the Nagumo boundary condition. An application to the fixed point theory of nonexpansive operators $F: \Omega \rightarrow X$ satisfying $F(\partial \Omega) \subset \Omega$ is given.
\end{abstract}

1. Introduction and main result. Denote by $X$ a real Banach space with norm $\|\cdot\|$, by $X^{*}$ the dual space of $X$, and by $J: X \rightarrow 2^{X^{*}}$ the duality mapping which is defined by

$$
J(x)=\left\{x^{*} \in X^{*} \mid x^{*}(x)=\|x\|^{2}=\left\|x^{*}\right\|^{2}\right\} .
$$

For each $x$ and $y$ in $X$ we put

$$
\begin{aligned}
& \langle x, y\rangle_{+}=\sup \left\{y^{*}(x) \mid y^{*} \in J(y)\right\}, \\
& \langle x, y\rangle_{-}=\inf \left\{y^{*}(x) \mid y^{*} \in J(y)\right\} .
\end{aligned}
$$

Let $\Omega$ be a nonempty closed bounded and starshaped subset of $X$ of positive diameter $L$. For $x \in X$, set

$$
d(x, \Omega)=\inf \{\|x-y\| y \in \Omega\} .
$$

Denote by $\mathfrak{T}$ the set of all (not necessarily linear) continuous operators $A$ : $\Omega \rightarrow X$ which are bounded, $\sup _{\Omega}\|A x\|<+\infty$, which are accretive, that is $\langle A x-A y, x-y\rangle_{-} \geqslant 0$ for all $x, y \in \Omega$, and which satisfy the Nagumo boundary condition [13], that is

$$
\lim _{h \rightarrow 0^{+}} h^{-1} d(x-h A x, \Omega)=0
$$

for each $x \in \Omega$.

$\Re$ is made into a complete metric space by defining [12, p. 246]

$$
\rho(A, B)=\sup \{\|A x-B x\| x \in \Omega\}, \quad A, B \in \Re \text {. }
$$

If $\Omega$ is convex, $\mathscr{T}$ is a convex cone.

Let $\Re$ be the subset of all $A \in \mathfrak{N}$ which are strongly accretive, that is

$$
\langle A x-A y, x-y\rangle_{-} \geqslant q_{A}\|x-y\|^{2}
$$

Received by the editors December 17, 1977 and, in revised form, October 12, 1978. AMS (MOS) subject classifications (1970). Primary 34G05, 47H10; Secondary 47H05.

$K e y$ words and phrases. Differential equation, asymptotic equilibrium, contraction semigroup, nonexpansive mapping, fixed point, Baire category, residual set, generic property, accretive operator, Nagumo boundary condition. 
for all $x, y \in \Omega\left(q_{A}>0\right)$. It is easy to see that $\Re$ is dense in $\mathscr{N}$. In fact, if $A \in \Re, \varepsilon>0$, and $z \in \Omega$ is such that for any $x \in \Omega$ the line segment $\alpha x+(1-\alpha) z, 0<\alpha<1$, is contained in $\Omega$, then the operator $B: \Omega \rightarrow X$, defined by

$$
B x=A x+(2 L)^{-1} \varepsilon(x-z),
$$

is in $\mathscr{T}$ and satisfies $\rho(A, B)<\varepsilon$. Clearly $B$ is continuous, bounded and strongly accretive. In addition, if $h q<1\left(h>0, q=(2 L)^{-1} \varepsilon\right)$, we have

$$
\begin{aligned}
d(x-h B x, \Omega) & =d(x-h A x-h q(x-z)-z+z, \Omega) \\
& =(1-h q) d\left(x-z-\frac{h}{1-h q} A x, \frac{\Omega-z}{1-h q}\right)
\end{aligned}
$$

from which, since $\Omega-z$ is starshaped with respect to the origin, we obtain

$$
d(x-h B x, \Omega)<(1-h q) d\left(x-z-\frac{h}{1-h q} A x, \Omega-z\right) .
$$

Dividing both sides of the latter inequality by $h>0$ and letting $h \rightarrow 0$ we have that $B$ satisfies the Nagumo boundary condition, and so $B \in \Re$.

For $A \in \mathscr{T}$ and $x \in \Omega$ consider the Cauchy problem

$$
u^{\prime}+A u=0, \quad u(0)=x
$$

(where the prime is, as usual, differentiation with respect to $t$ ). By a solution of (1) we mean any continuously differentiable function $S_{A}(\cdot) x:[0,+\infty) \rightarrow \Omega$ satisfying (1) for all $t>0$. The following theorems are due to Martin [11] and Vidossich [16], respectively.

TheOREM I. For each $A \in \mathfrak{T}$ and each $x \in \Omega$, the problem (1) has a unique solution.

Let $A \in \mathfrak{N}$. A point $\omega_{A} \in \Omega$, such that for every $x \in \Omega$ we have $\lim _{t \rightarrow+\infty} S(t) x=\omega_{A}$, is called an asymptotic equilibrium of $A$.

THEOREM II. Each $A \in \Re$ has a unique asymptotic equilibrium.

For each $A \in \mathfrak{N}$, the family of maps $S_{A}(t): x \rightarrow S_{A}(t) x$ is a continuous one-parameter semigroup of nonexpansive transformations of $\Omega$ into itself. By Theorem II, each $A \in \Re$ has a unique asymptotic equilibrium. This is no longer true, in general, whenever $A \in \mathfrak{R}$. As a simple example, the map $A(x, y)=(-y, x)$, restricted to the unit ball of $R^{2}$, has no asymptotic equilibrium. However such a situation is to be considered quite exceptional in view of the following.

TheOREM 1. Let $\mathfrak{T}_{0}$ be the subset of all those $A \in \mathfrak{T}$ which have a unique asymptotic equilibrium $\omega_{A}$. Then $\mathfrak{T}_{0}$ is a residual set in $\Re$.

From Theorem 1 we obtain immediately:

COROLlary 1. The subset of all those $A \in \mathfrak{N}$ such that $A^{-1}(0)$ consists of a unique point is a residual set in $\Re$. 
For related results on the existence of zeros of accretive operators, see [4], [10], [14], [16].

COROLlary 2. The subset of all those $A \in \mathfrak{T}$ such that $u^{\prime}+A u=0$ has at least one nonconstant periodic solution or at least two (different) constant solutions is of Baire first category in $\mathfrak{N}$.

2. Application to fixed point theory. Theorem 1 can be applied to the fixed point theory of nonexpansive operators. In this section we assume that $\Omega$ is also convex. Let $\mathscr{F}=\{F: \Omega \rightarrow X \mid F$ nonexpansive, $F(\partial \Omega) \subset \Omega\}$. Here $\partial \Omega$ denotes the boundary of $\Omega$. $\mathscr{F}$ is made into a (complete) metric space if we define

$$
\sigma(F, G)=\sup \{\|F x-G x\| x \in \Omega\} \quad(F, G \in \mathscr{F}) .
$$

Observe that $\mathscr{F}$ is convex.

The following theorem shows that certain recent extensions [5], [9] of the classical Browder-Gödhe-Kirk fixed point theorem [1], [6], [8] obtained within the framework of spaces with normal structure, still remain valid for most mappings in a general Banach space.

THEOREM 2. Let $\mathscr{F}_{0}$ be the subset of all $F \in \mathscr{F}$ which have a unique fixed point. Then $\mathscr{F}_{0}$ is a residual set in $\mathscr{F}_{\text {. }}$

This theorem generalizes a theorem (proved by Vidossich [16] by a quite different technique) ensuring that most nonexpansive mappings from $\Omega$ into itself have a unique fixed point. A result of constructive type stating that, for most nonexpansive self-mappings on $\Omega$ the sequence of successive approximations actually does converge, is proved in [2]. We wonder whether a similar constructive result could be obtained for maps $F \in \mathscr{F}$. (A partial answer is furnished by Theorem 3.)

However the theorem of [2] has a counterpart in the theory of ordinary differential equations in an infinite dimensional Banach space. In fact it is proved in [3] that: In the Banach space $\mathbb{Q}$ of all continuous and bounded vector fields $f:[0,1] \times U_{r} \rightarrow X$ where $U_{r}=\left\{x \in X \mid\left\|x-x_{0}\right\|<r\right\}(r>0)$, with the supremum norm, the subset of all $f \in \mathbb{Q}$ such that the Peano-Picard successive approximations for $u^{\prime}=f(t, u), u(0)=x_{0}$ converge, is a residual set in $\mathbb{Q}$.

Theorem 3. Suppose that $X$ is a Hilbert space. For $y \in X$ let Py be the projection of $y$ on the set $\Omega \subset X$. Let $\mathcal{F}_{1}$ be the subset of all $F \in F_{\text {such }}$ that the sequence of the successive approximations $\left\{(P F)^{n} x\right\}$ converges to the unique fixed point of $F$, for every starting point $x \in \Omega$. Then $\mathscr{F}_{1}$ is a residual set in $\mathscr{F}$.

3. Proofs. Let $\mathcal{X}$ be a metric space. We denote by $V(h, \delta)$ the open ball in $\mathcal{X}$ with center $h$ and positive radius $\delta$. 
Proof of Theorem 1. Claim 1. Let $B \in \Re$ and $\varepsilon>0$ be given. Then there exists $\delta_{B}(\varepsilon)>0$ such that for each $A \in V\left(B, \delta_{B}(\varepsilon)\right)$ we have

$$
\left\|S_{B}(t) x-S_{A}(t) x\right\| \leqslant \varepsilon
$$

for every $x \in \Omega$ and all $t \geqslant 0$.

Choose $\delta_{B}(\varepsilon)$ satisfying $0<\delta_{B}(\varepsilon) \leqslant \varepsilon^{2} q_{B} / L$ where $q_{B}>0$ corresponds to $B \in \mathcal{N}$ and $L>0$ is the diameter of $\Omega$. Let $A \in V\left(B, \delta_{B}(\varepsilon)\right)$, and let $x \in \Omega$ be arbitrary. Using Kato's lemma [7], for almost all $t \geqslant 0$ we have

$$
\begin{aligned}
\frac{d}{d t} \frac{1}{2} \| & S_{B}(t) x-S_{A}(t) x \|^{2} \\
= & \left\langle-B S_{B}(t) x+A S_{A}(t) x, S_{B}(t) x-S_{A}(t) x\right\rangle_{+} \\
= & \left\langle-B S_{B}(t) x+B S_{A}(t) x-B S_{A}(t) x+A S_{A}(t) x, S_{B}(t) x-S_{A}(t) x\right\rangle_{+} \\
\leqslant & \left\langle-B S_{B}(t) x+B S_{A}(t) x, S_{B}(t) x-S_{A}(t) x\right\rangle_{+} \\
& +\left\|B S_{A}(t) x-A S_{A}(t) x\right\| \cdot\left\|S_{B}(t) x-S_{A}(t) x\right\| \\
< & -\left\langle B S_{B}(t) x-B S_{A}(t) x, S_{B}(t) x-S_{A}(t) x\right\rangle_{-}+\delta_{B}(\varepsilon) L \\
< & -q_{B}\left\|S_{B}(t) x-S_{A}(t) x\right\|^{2}+\delta_{B}(\varepsilon) L .
\end{aligned}
$$

Solving this differential inequality and noting that $S_{A}(0) x=S_{B}(0) x=x$, furnishes $\left\|S_{B}(t) x-S_{A}(t) x\right\| \leqslant \varepsilon$ for all $t \geqslant 0$, and Claim 1 is true.

Claim 2. We have

$$
\Re_{*}=\bigcap_{n=1}^{\infty} \bigcup_{B \in \Re} V\left(B, \delta_{B}\left(\frac{1}{n}\right)\right) \subset \Re_{0} .
$$

Let $A \in \mathfrak{T}_{*}$. Then, there exists a sequence $\left\{B_{n}\right\} \subset \Re$ such that $A \in$ $V\left(B_{n}, \delta_{B_{n}}(1 / n)\right)$ for $n=1,2, \ldots$, and so

$$
\left\|S_{A}(t) x-S_{B_{n}}(t) x\right\| \leqslant 1 / n
$$

for all $t>0, n=1,2, \ldots$ The corresponding sequence of asymptotic equilibria $\left\{\omega_{B_{n}}\right\}$ is Cauchy. For, given $\varepsilon>0$, if $n, m>4 / \varepsilon$ and $t$ is sufficiently large we have

$$
\begin{aligned}
\left\|\omega_{B_{n}}-\omega_{B_{m}}\right\|< & \left\|\omega_{B_{n}}-S_{B_{n}}(t) x\right\|+\left\|S_{B_{n}}(t) x-S_{A}(t) x\right\| \\
& +\left\|S_{A}(t) x-S_{B_{m}}(t) x\right\|+\left\|S_{B_{m}}(t) x-\omega_{m}\right\| \\
& <\frac{\varepsilon}{4}+\frac{1}{n}+\frac{1}{m}+\frac{\varepsilon}{4}<\varepsilon .
\end{aligned}
$$

Let $\lim _{n \rightarrow+\infty} \omega_{B_{n}}=\omega$. Let $x \in \Omega$ and let $S_{A}(\cdot) x$ be the corresponding solution of (1). Since for $n$ and $t$ large enough we have

$$
\begin{aligned}
\left\|S_{A}(t) x-\omega\right\| & <\left\|S_{A}(t) x-S_{B_{n}}(t) x\right\|+\left\|S_{B_{n}}(t) x-\omega_{B_{n}}\right\|+\left\|\omega_{B_{n}}-\omega\right\| \\
& <\frac{1}{n}+\frac{\varepsilon}{3}+\frac{\varepsilon}{3},
\end{aligned}
$$

if follows that $\lim _{t \rightarrow+\infty} S_{A}(t) x=\omega$. This shows that $\omega_{A}=\omega$ is an asymptotic equilibrium of $A$. The uniqueness of $\omega_{A}$ is obvious, hence $A \in \Re_{0}$. 
Clearly $\mathfrak{K}_{*}$ is dense in $\mathscr{N}$ since $\mathscr{\Re} \subset \mathfrak{K}_{*}$. Thus to complete the proof it suffices to observe that $\mathfrak{K}_{*}$, as a dense $G_{\delta}$-set in the Baire space $\mathscr{K}$, is residual. The same is true a fortiori for $\mathfrak{T}_{0}$, since $\mathfrak{K}_{0} \supset \Re_{*}$.

COROLlaRy 3. Let $\mathcal{L}$ be a (nonempty) closed subspace of $\Re$. Let $\mathcal{R} \cap$ be dense in $\mathcal{L}$. Then the subset $\mathfrak{L}_{0}$ of all $A \in \mathcal{L}$, such that $A^{-1}(0)$ consists of a unique point, is a residual set in $\mathcal{L}$.

Proof. By the preceding argument we prove that

$$
\mathcal{E}_{*}=\bigcap_{n=1}^{\infty} \bigcup_{B \in \mathcal{E}_{\cap} \pi} V\left(B, \delta_{B}\left(\frac{1}{n}\right)\right)
$$

is a residual set in $\mathcal{L}$. Here $V$ stands for an open ball in $\mathcal{E}$. The statement follows from the inclusion $e_{*} \subset \mathcal{L}_{0}$.

Proof of Theorem 2. The function $\Lambda: F \rightarrow I-F, I$ the identity, is a bijection of $\mathscr{F}$ onto $\tilde{F}=\Lambda(\mathscr{F})$. For each $F \in \mathscr{F}, \Lambda(F)$ is continuous, bounded and accretive; furthermore the fact that $F(\partial \Omega) \subset \Omega$ implies that $\Lambda(F)$ satisfies the Nagumo condition. Thus $\tilde{\mathscr{F}} \subset \mathfrak{N}$ (if, of course, in the definition of $\mathscr{T}$ we assume $\Omega$ to be convex). By the isometry between $\mathscr{F}$ and $\tilde{\mathscr{F}}$ we deduce that $\tilde{\mathscr{F}}$ is a closed subspace of $\Re$. When $G$ is a strict contraction, $\Lambda(G)$ is strongly accretive, hence $\Lambda(G) \in \tilde{\mathscr{F}} \cap \Re$ and the latter set is dense in $\tilde{\mathscr{F}}$ since strict contractions are dense in $\mathscr{F}$. By Corollary 3 the subset $\tilde{\mathscr{F}}_{0}$ of all $\Lambda(F)$ with a unique zero is residual in $\tilde{\mathscr{F}}$. By the isometry, $\mathscr{F}_{0}=\Lambda^{-1}\left(\tilde{\mathscr{F}}_{0}\right)$ is residual in $\mathscr{F}$ and, since any $F \in \mathscr{F}_{0}$ has a unique fixed point, the proof is complete.

Proof of Theorem 3. Let $\mathcal{G}$ be the subset of all strict contractions $G \in \mathscr{F}$. When $G \in \mathcal{G}$ the composition $P G$ is a strict contraction (with the same Lipschitz constant of $G$, say $q_{G}$ ) and maps $\Omega$ into itself, hence it has a unique fixed point $x_{G}$. The boundary condition $G(\partial \Omega) \subset \Omega$ implies that $x_{G}$ is also the (unique) fixed point of $G$. For fixed $x_{0} \in \Omega$ and $F \in \mathcal{F}$ the map $G_{\alpha} x=\alpha x_{0}$ $+(1-\alpha) F x, 0<\alpha<1, x \in \Omega$ is in $\mathcal{G}$ and when $\alpha \rightarrow 0$ we have $G_{\alpha} \rightarrow F$. This proves that $\mathcal{G}$ is dense in $\mathscr{F}$. Let $G \in \mathcal{G}$ and $\varepsilon>0$ be arbitrary. Take $0<\delta(\varepsilon)<\left(1-q_{G}\right) \varepsilon$. For any $F \in V\left(G, \delta_{G}(\varepsilon)\right)$ and any $x \in \Omega$, we have that

$$
\begin{aligned}
\|(P G)^{2} x & -(P F)^{2} x \| \\
& <\|(P G)(P G) x-(P G)(P F) x\|+\|(P G)(P F) x-(P F)(P F) x\| \\
& <q_{G} \delta_{G}(\varepsilon)+\delta_{G}(\varepsilon)=\left(1+q_{G}\right) \delta_{G}(\varepsilon),
\end{aligned}
$$

and easily, using induction, that $\left\|(P G)^{n} x-(P F)^{n} x\right\|<\varepsilon$ for all $n=$ $1,2, \ldots$ Then the set

$$
\mathscr{F}_{*}=\bigcap_{n=1}^{\infty} \bigcup_{G \in \mathcal{G}} V\left(G, \delta_{G}\left(\frac{1}{n}\right)\right)
$$

is residual in $\mathscr{F}$ and, as in Claim 2, it is proved that for each $F \in \mathscr{F}_{*}$ and for each $x \in \Omega$ the sequence $\left\{(P F)^{n} x\right\}$ converges to a limit $x_{F}$. Since $F(\partial \Omega) \subset \Omega$ 
we have the result that $F x_{F}=x_{F}$. The uniqueness is trivial. Since $\mathscr{F}_{*} \subset \mathscr{F}_{1}$, the proof is complete.

Simple examples show (even in the finite dimensional case) that $\mathscr{F}_{\backslash} \mathscr{F}_{1}$ can be nonempty.

4. Further results. The following two theorems are concerned with the continuous dependence of the asymptotic equilibria, or fixed points, upon the data. The proofs are omitted since they run as for Theorem 2 of [3].

TheOREM 4. There exists a Baire first category set $\mathscr{K} \subset \mathfrak{T}$ such that the map $\varphi: \mathscr{K} \backslash \mathcal{K} \rightarrow \Omega$ given by $\varphi(A)=\omega_{A}$ is well defined and continuous.

For $F \in \mathscr{F}$ denote by $x_{F}$ the fixed point of $F$, if it exists and is unique.

THEOREM 5. There exists a Baire first category set $\mathscr{P} \subset \mathcal{F}$ such that the map $\psi: \mathcal{F} \backslash \mathscr{P} \rightarrow \Omega$ given by $\psi(F)=x_{F}$ is well defined and continuous.

In the next theorem we consider the structure of the subset $\mathfrak{R}_{*}$ of the convex cone $\Re$.

THEOREM 6. $\Re_{*} \cup\{0\}$ is a convex cone in $\Re$.

Proof. Observe that for each $\varepsilon>0$ and $B \in \Re$ the statement of Claim 1 is certainly satisfied if we take $\delta_{B}(\varepsilon)=\varepsilon^{2} q_{B} / 2 L$.

It is easy to see that if $B \in \mathcal{N}$ with constant $q_{B}$, then $\alpha B \in \Re$ with constant $\alpha q_{B}(\alpha>0)$ and so, if we choose $\delta_{\alpha B}(\varepsilon)=\alpha \delta_{B}(\varepsilon)$, the conclusion of Claim 1 holds.

Let $A \in \Re_{*}$ and $\alpha>0$. There is a sequence $\left\{B_{n}\right\} \subset \Re$ such that $A \in$ $V\left(B_{n}, \delta_{B_{n}}(1 / n)\right), n=1,2, \ldots$ Thus

$$
\alpha A \in V\left(\alpha B_{n}, \alpha \delta_{B_{n}}\left(\frac{1}{n}\right)\right)=V\left(\alpha B_{n}, \delta_{\alpha B_{n}}\left(\frac{1}{n}\right)\right), \quad n=1,2, \ldots,
$$

implies that $\alpha A \in \mathscr{N}_{*}$. The case $\alpha=0$ is trivial.

An easy calculation shows that if $B, \tilde{B} \in \mathcal{K}$ with constants $q_{B}, q_{\tilde{B}}$, respectively, and $\alpha, \beta>0, \alpha+\beta=1$, then $\alpha B+\beta \tilde{B} \in \Re$ with constant

$$
q_{\alpha B+\beta \tilde{B}}=\alpha q_{B}+\beta q_{\tilde{B}} \text {. }
$$

From this we have that the conclusion of Claim 1 is satisfied for

$$
\delta_{\alpha B+\beta \tilde{B}}(\varepsilon)=\alpha \delta_{B}(\varepsilon)+\beta \delta_{\tilde{B}}(\varepsilon) .
$$

Let $A, \tilde{A} \in \mathfrak{N}_{*}$ and $\alpha, \beta>0, \alpha+\beta=1$. There are sequences $\left\{B_{n}\right\}$, $\left\{\tilde{B}_{n}\right\} \subset \mathscr{T}$ such that $A \in V\left(B_{n}, \delta_{B_{n}}(1 / n)\right), \tilde{A} \in V\left(\tilde{B}_{n}, \delta_{\tilde{B}_{n}}(1 / n)\right), \quad n=$ $1,2, \ldots$ Then

$$
\begin{aligned}
\alpha A+\beta \tilde{A} \in V\left(\alpha B_{n}, \alpha \delta_{B_{n}}\left(\frac{1}{n}\right)\right)+V\left(\beta \tilde{B}_{n}, \beta \delta_{\tilde{B}_{n}}\left(\frac{1}{n}\right)\right) \\
\quad=V\left(\alpha B_{n}+\beta \tilde{B}_{n}, \delta_{\alpha B_{n}}+\beta \tilde{B}_{n}(1 / n)\right), \quad n=1,2, \ldots,
\end{aligned}
$$

implies that $\alpha A+\beta \tilde{A} \in \mathfrak{T}_{*}$. This completes the proof. 


\section{REFERENCES}

1. F. E. Browder, Nonexpansive nonlinear operators in a Banach space, Proc. Nat. Acad. Sci. U.S.A. 34 (1965), 1041-1044.

2. F. S. De Blasi and J. Myjak, Sur la convergence des approximations successives pour les contractions non linéaires dans un espace de Banach, C. R. Acad. Sci. Paris Sér. A-B 4 (1976), 185-188.

3. Generic properties of differential equations in Banach space, Bull. Acad. Polon. Sci. Sér. Math. Astronom. Phys. 26 (1978), 287-292.

4. K. Deimling, Zeros of accretive operators, Manuscripta Math. 13 (1974), 365-374.

5. J. Gatica and W. A. Kirk, Fixed point theorems for Lipschitzian pseudocontractive mappings, Proc. Amer. Math. Soc. 36 (1972), 111-115.

6. D. Gödhe, Zum Prinzip der kontraktiven Abbildung, Math. Nachr. 30 (1966), 251-258.

7. T. Kato, Nonlinear semigroups in Banach space, J. Math. Soc. Japan 19 (1967), 493-507.

8. W. A. Kirk, A fixed point theorem for mappings which do not increase distances, Amer. Math. Monthly 72 (1965), 1004-1006.

9. pings, Pacific J. Math. 38 (1971), 89-94.

10. V. Lakshmikantham and S. Leela, On the existence of zeros of Lyapunov-monotone operators, University of Texas, Arlington, Technical Report No. 19, 1975.

11. R. H. Martin, Jr., Differential equations on closed subsets of a Banach space, Trans. Amer. Math. Soc. 179 (1973), 399-414.

12. Nonlinear operators and differential equations in Banach spaces, Wiley-Interscience, New York, 1976.

13. N. Nagumo, Über die Lage der Intergralkurven gewöhnlicher differential Gleichungen, Proc. Phys. Math. Soc. Japan 24 (1942), 551-559.

14. S. Reich, On fixed point theorems obtained from existence theorems for differential equations, J. Math. Anal. Appl. 54 (1976), 26-36.

15. G. Vidossich, Existence, uniqueness and approximation of fixed points as a generic property, Bol. Soc. Brasil. Mat. 5 (1974), 17-29.

16. How to get zeros of nonlinear operators using the theory of ordinary differential equations, Atas Semana Analise Funcional, São Paulo, 1973.

Universitì degli Studi, Istituto Matematico “U. Din”, Viale Morgagni 67/A, 50134 Firenze, Italy

Instytut Matematyxi AGH, AL. Mickiewicza 30, 30-059 Kraków, Poland 